\title{
Usefulness of Scintigraphy to Evaluate Adrenal Tumors
}

\author{
Tadanao Harigae ${ }^{\mathrm{a}, \mathrm{b}}$, Naonori Tsuda ${ }^{\mathrm{a}, \mathrm{b}}$, Hiroki Hashimoto ${ }^{\mathrm{a}}$, \\ Hidekatsu Yanai ${ }^{\mathrm{a}, \mathrm{c}}$
}

\section{To the Editor}

If we find adrenal tumors in patients, how should we do? We should examine to make clear whether the tumor is hormoneproducing or not, and whether the tumor is benign or malignant. To make sure that the tumor is malignant or not, what can we do? The diameter of the tumor can be useful. Tumor size at presentation (mean diameter at diagnosis $>10 \mathrm{~cm}$ ) has been reported to be the most important indicator of malignancy [1]. Another review article described that a functioning adrenal carcinoma should be considered when an adrenal mass measures more than $5 \mathrm{~cm}$ in diameter [2]. However, in the previous study which examined adrenal tumors of 57 patients who underwent adrenalectomy, the mean diameter of the resected primary adenocarcinomas was 3.0 and $4.5 \mathrm{~cm}$, respectively [3]. A very recent study reported that tumor size $\geq 40 \mathrm{~mm}$ was significantly associated with malignancy [4]. In addition to tumor size, elevated serum dehydroepiandrosterone sulphate (DHEAS) can predict malignancy [4].

After the rule-out of the possibility for malignancy using CT, MRI and measurement of serum DHEAS, we examine blood hormone levels. Plasma renin and aldosterone levels, serum $\mathrm{ACTH}$, cortisol, and catecholamine levels (dopamine, noradrenaline and adrenaline) are measured. If cortisol increased and ACTH decreased, Cushing's syndrome was suspected. In Cushing's syndrome, diurnal variation of cortisol disappeared and urinary cortisol level increased ( $>80 \mu \mathrm{g}$ /day). I-adosterol scintigraphy can confirm the existence of cortisol-producing tumor (Fig. 1). A patient B was referred to our hospital due to resistant hypertension. Serum noradrenaline level was high (576 $\mathrm{pg} / \mathrm{mL}$; normal range, 100 - $450 \mathrm{pg} / \mathrm{mL}$ ); however, daily urinary metanephrine and normetanephrine levels were within normal range. Therefore, we performed I-metaiodobenzylguanidine (MIBG) scintigraphy, and could detect the uptake of I-MIBG in adrenal tumor (Fig. 1), which made us diagnose this patient.

To evaluate functioning (hormone-producing) adrenal tumors, in addition to endocrinological tests, an appropriate scintigraphy may be useful.

\section{Conflict of Interest}

The authors declare that they have no conflict of interest concerning this article.

\section{References}

1. Allolio B, Hahner S, Weismann D, Fassnacht M. Management of adrenocortical carcinoma. Clin Endocrinol (Oxf). 2004;60(3):273-287.

2. Iihara M, Obara T. [Diagnosis and surgical treatment of adrenal tumors]. Nihon Geka Gakkai Zasshi. 2005;106(8):479-483.

3. Linos DA, Stylopoulos N, Raptis SA. Adrenaloma: a call for more aggressive management. World J Surg. 1996;20(7):788-792; discussion 792-783.

4. Foo E, Turner R, Wang KC, Aniss A, Gill AJ, Sidhu S, Clifton-Bligh $\mathrm{R}$, et al. Predicting malignancy in adrenal incidentaloma and evaluation of a novel risk stratification algorithm. ANZ J Surg. 2017. 


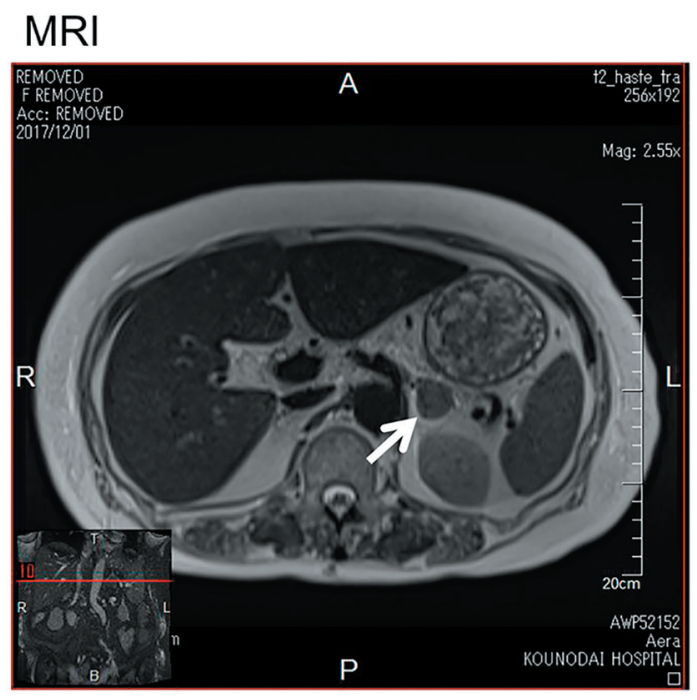

\section{l-adosterol scintigraphy a}

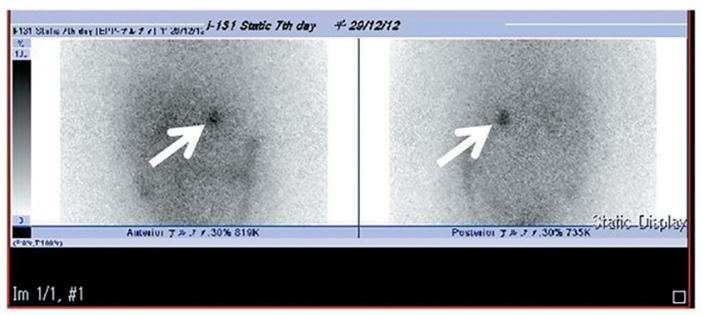

MRI

I-MIBG scintigraphy

b
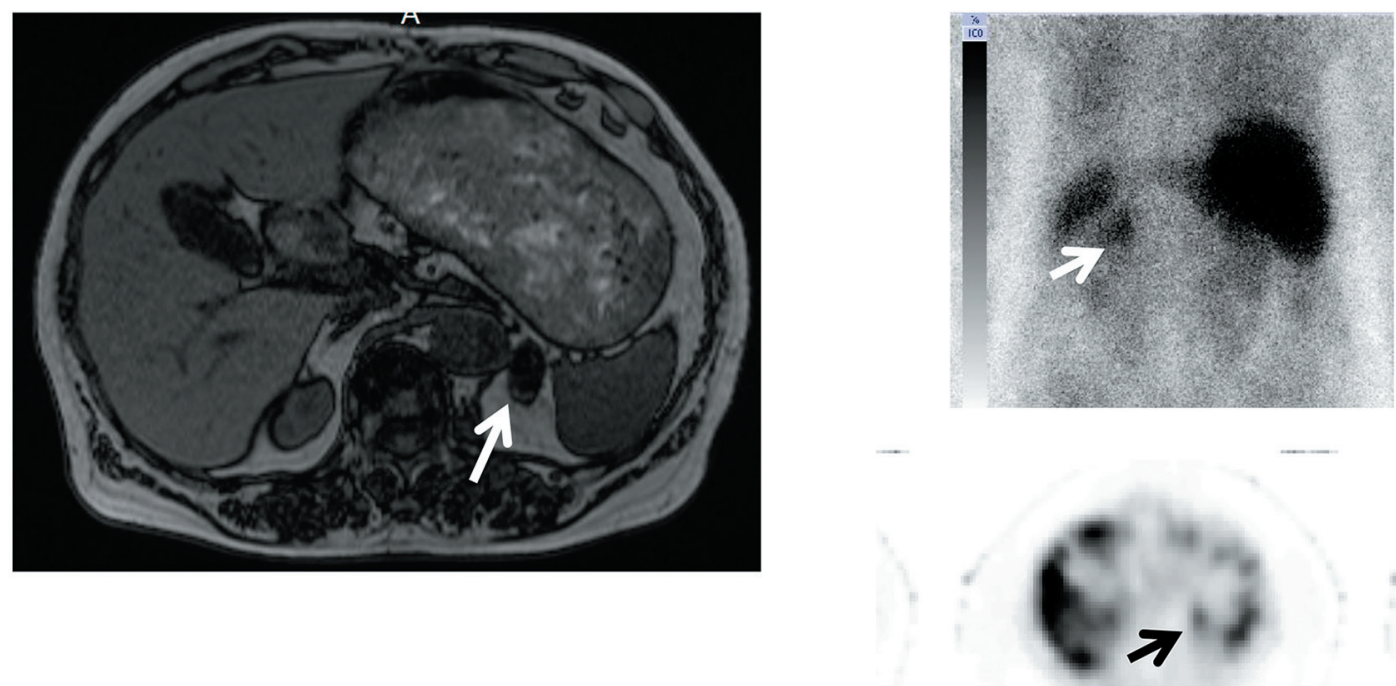

Figure 1. MRI and scintigraphy of patients with Cushing' syndrome (a) and pheochromocytoma (b). 